\title{
SYNTHESIS OF 10H-PHENOTHIAZINE AND 4H-1,4-BENZOTHIAZINE SULFONES
}

\author{
Leby Thomas, Archana Gupta and Vandana Gupta* \\ Department of Chemistry, University of Rajasthan, Jaipur-302004 (India)
}

\begin{abstract}
Synthesis of phenothiazine and 4H-1,4-benzothiazine sulfones is reported by the oxidation of phenothiazines and $4 \mathrm{H}-1,4$-benzothiazines respectively by $30 \%$ hydrogen peroxide in glacial acetic acid. The purity of the compounds has been checked by thin layer chromatography using various non-aqueous solvent systems. IR and NMR spectral studies have also been included.

\section{Introduction}

10H-Phenothiazine and 4H-1,4-benzothiazine sulfones constitute an important class of heterocyclic compounds which have been reported to find a number of applications in medicine (1-13) and industry (14-15). Phenothiazine and 1,4-benzothiazine sulfones were obtained in quantitative yield by the oxidation of phenothiazines and benzothiazines respectively with hydrogen peroxide (16).

\section{Results and Discussion}

The title sulfones have been synthesized by the oxidation of phenothiazines and 1,4-benzothiazines. Phenothiazines were prepared by the Smiles rearrangement of 2-amino-3methyl / 5-methylbenzenethiols with o-halonitrobenzenes and 1,4-benzothiazines were prepared by the condensation and oxidative cyclization of 2-amino-3-methyl / 5-methyl benzenethiols with $\beta$-diketones / $\beta$-ketoesters in dimethylsulfoxide reported elsewhere (17-19).

Phenothiazine-5,5-dioxides (Scheme 1, II a-j) and 4H-1,4-benzothiazine-1, 1-dioxides (Scheme 2, IV a-h) have been prepared by treating phenothiazines and 1,4-benzothiazines respectively with $30 \%$ hydrogen peroxide in glacial acetic acid.

The IR spectra of phenothiazine and 4H-1,4-benzothiazine sulfones have been recorded both in potassium bromide pellets and in chloroform solution. All the synthesized phenothiazine and $4 \mathrm{H}-1,4$-benzothiazine sulfones exhibit a sharp intense peak in the region $1390-1300 \mathrm{~cm}^{-1}$ and $1390-1310 \mathrm{~cm}^{-1}$ respectively in chloroform which can be ascribed to the 


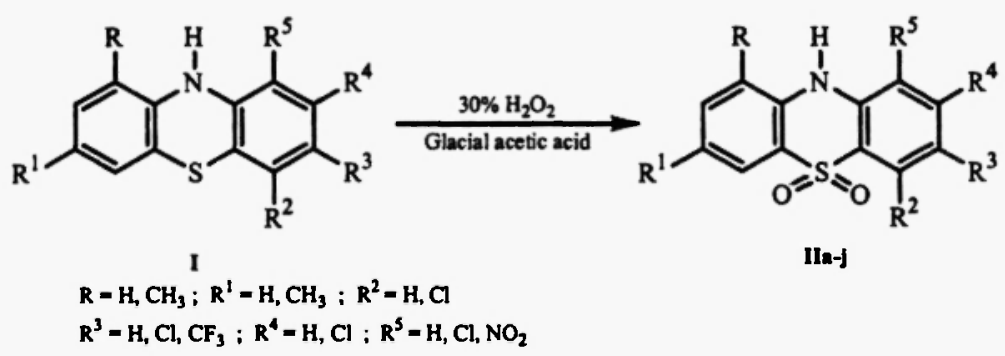

Scheme 1<smiles>[R]C(=O)C1=C(C)Nc2c([R])cc([R])cc2S1</smiles><smiles>[R]C(=O)C1=C(C)Nc2c([R])cc([R])cc2S1(=O)=O</smiles>

$\mathrm{R}-\mathrm{H}_{1} \mathrm{CH}_{3} ; \mathrm{R}^{\prime}-\mathrm{H}_{1} \mathrm{CH}_{3} ;$

$\mathrm{R}^{2}-\mathrm{C}_{6} \mathrm{H}_{6}-\mathrm{Br}(\mathrm{m}), \mathrm{C}_{6} \mathrm{H}_{4}-\mathrm{CH}_{3}(\mathrm{~m}), \mathrm{C}_{6} \mathrm{H}_{4}-\mathrm{OC}_{2} \mathrm{H}_{5}(\mathrm{p}), \mathrm{C}_{6} \mathrm{H}_{4}-\mathrm{C}_{2} \mathrm{H}_{5}(\mathrm{p})$

Scheme 2

asymmetric stretching $\left(v_{3}\right)$ mode of sulphonyl group which in the solid state splits into three bands in the region 1420-1330 cm phenothiazine sulfones and in benzothiazine sulfones it appears in the region $1420-1390 \mathrm{~cm}^{-1}$, $1330-1300 \mathrm{~cm}^{-1}$ and $1270-1200 \mathrm{~cm}^{-1}$. The symmetrical stretching vibrations $\mathrm{v}_{1}$ of phenothiazine and $4 \mathrm{H}-1$,4-benzothiazine sulfones give rise to a doublet in potassium bromide pellets in the region $1180-1100 \mathrm{~cm}^{-1}$ and $1180-1050 \mathrm{~cm}^{-1}$ whereas in solution it appears in the region $1180-1080 \mathrm{~cm}^{-1}$ and $1180-1100 \mathrm{~cm}^{-1}$ respectively. The band appearing in the region $600-500 \mathrm{~cm}^{-1}$ and $590-510 \mathrm{~cm}^{-1}$ in phenothiazine and benzothiazine sulfones respectively can be attributed to bending vibrations $\left(v_{2}\right)$.

The N-H stretching frequency appears in the region $3430-3305 \mathrm{~cm}^{-1}$ and $3420-3315 \mathrm{~cm}^{-1}$ in phenothiazine and $4 \mathrm{H}-1$,4-benzothiazine sulfones respectively. A sharp band in the region $1700-1600 \mathrm{~cm}^{-1}$ appears due to $C=0$ stretching vibrations in 4H-1,4-benzothiazine sulfones. The asymmetric and symmetric stretching vibrations of methyl group in phenothiazine sulfones occur in the region 2960-2920 $\mathrm{cm}^{-1}$ and $2890-2850 \mathrm{~cm}^{-1}$ which in 4H-1,4-benzothiazine sulfones occur in the region $2980-2930 \mathrm{~cm}^{-1}$ and2950-2860 $\mathrm{cm}^{-1}$. All the phenothiazine and benzothiazine sulfones exhibit a medium 
intensity band in the region $1080-1040 \mathrm{~cm}^{-1}$ and $1070-1030 \mathrm{~cm}^{-1}$ respectively due to C-S stretching vibrations.

The ' $\mathrm{H}$ NMR spectra of phenothiazine sulfones II $\mathbf{a}-\mathbf{j}$ and $4 \mathrm{H}-1,4-$ benzothiazine sulfones IV a-d exhibit a sharp peak in the region $\delta 9.926-9.352 \mathrm{ppm}$ and $\delta 10.30-9.70 \mathrm{ppm}$ respectively due to $\mathrm{N}-\mathrm{H}$ proton. The multiplets observed in the region $\delta 8.89-6.48 \mathrm{ppm}$ and $\delta$ 8.781-6.61 ppm respectively are attributed to the aromatic protons. Compounds II a,g,h exhibits a singlet in the region $\delta 3.663-2.663$ ppm due to $\mathrm{CH}_{3}$ protons at $\mathrm{C}_{9}$. Compounds II b and II c exhibit a singlet at $\delta 2.61 \mathrm{ppm}$ and $\delta 2.168 \mathrm{ppm}$ respectively due to $\mathrm{CH}_{3}$ protons at $\mathrm{C}_{1}$. A singlet in the region $\delta 3.402-1.934 \mathrm{ppm}$ is observed in compounds $\mathbf{I V} \mathbf{d - f}, \mathbf{i}, \mathbf{j}$ due to three protons of $\mathrm{CH}_{3}$ group at $\mathrm{C}_{7}$. Compounds IV a-d show resonance signal in the region $\delta 2.49-1.81 \mathrm{ppm}$ due to allylic protons at $\mathrm{C}_{3}$ and another singlet in the region $\delta 2.38-1.744$ ppm due to $\mathrm{CH}_{3}$ protons at $\mathrm{C}_{7}$. Compound IV b exhibits a singlet at $\delta 2.40 \mathrm{ppm}$ due to $\mathrm{CH}_{3}$ protons at meta position of benzoyl side chain at $\mathrm{C}_{2}$. Compound IV $\mathrm{c}$ exhibits a quartet and a triplet in the region $\delta 4.35-3.39 \mathrm{ppm}$ and $\delta 1.75-1.22 \mathrm{ppm}$ due to $\mathrm{CH}_{2}$ and $\mathrm{CH}_{3}$ protons of $\mathrm{OC}_{2} \mathrm{H}_{5}$ group at para position of benzoyl side chain at $\mathrm{C}_{2}$. The quartet and triplet observed in the region $\delta 3.202-2.63 \mathrm{ppm}$ and $\delta 1.24-1.01 \mathrm{ppm}$ in the compound IV d can be assigned to $\mathrm{C}_{2} \mathrm{H}_{5}$ group at para position of benzoyl side chain at $\mathrm{C}_{2}$.

\section{Experimental}

All the melting points are uncorrected. The purity of the compounds has been checked by thin layer chromatography using various non-aqueous solvents and characterized by spectral studies. The infrared spectra were recorded on FT IR spectrometer, MAGNA IR 550, NICOLET using potassium bromide discs and in chloroform solution. NMR spectra were recorded on FT NMR Bruker DRX-300 MHz in DMSO- $\mathrm{d}_{6}$ or $\mathrm{CDCl}_{3}$ containing TMS as an internal standard. Analytical data of newly synthesized compounds are summarized in Tables 1 and 2.

Preparation of phenothiazine sulfones (II a-j) and 4H-1,4-benzothiazine sulfones (IV a-h)

To a solution of 0.01 mole of phenothiazines or $4 \mathrm{H}-1,4$-benzothiazines in $15 \mathrm{ml}$ of glacial acetic acid, $5 \mathrm{ml}$ of $30 \%$ hydrogen peroxide was added and refluxed at $50-60^{\circ} \mathrm{C}$ for fifteen minutes. Heating was stopped and another lot of $5 \mathrm{ml}$ of $30 \%$ hydrogen peroxide was added. The solution was refluxed for four hours. A major portion of the solvent was then removed by distillation under reduced pressure and then the solution was poured into a beaker 


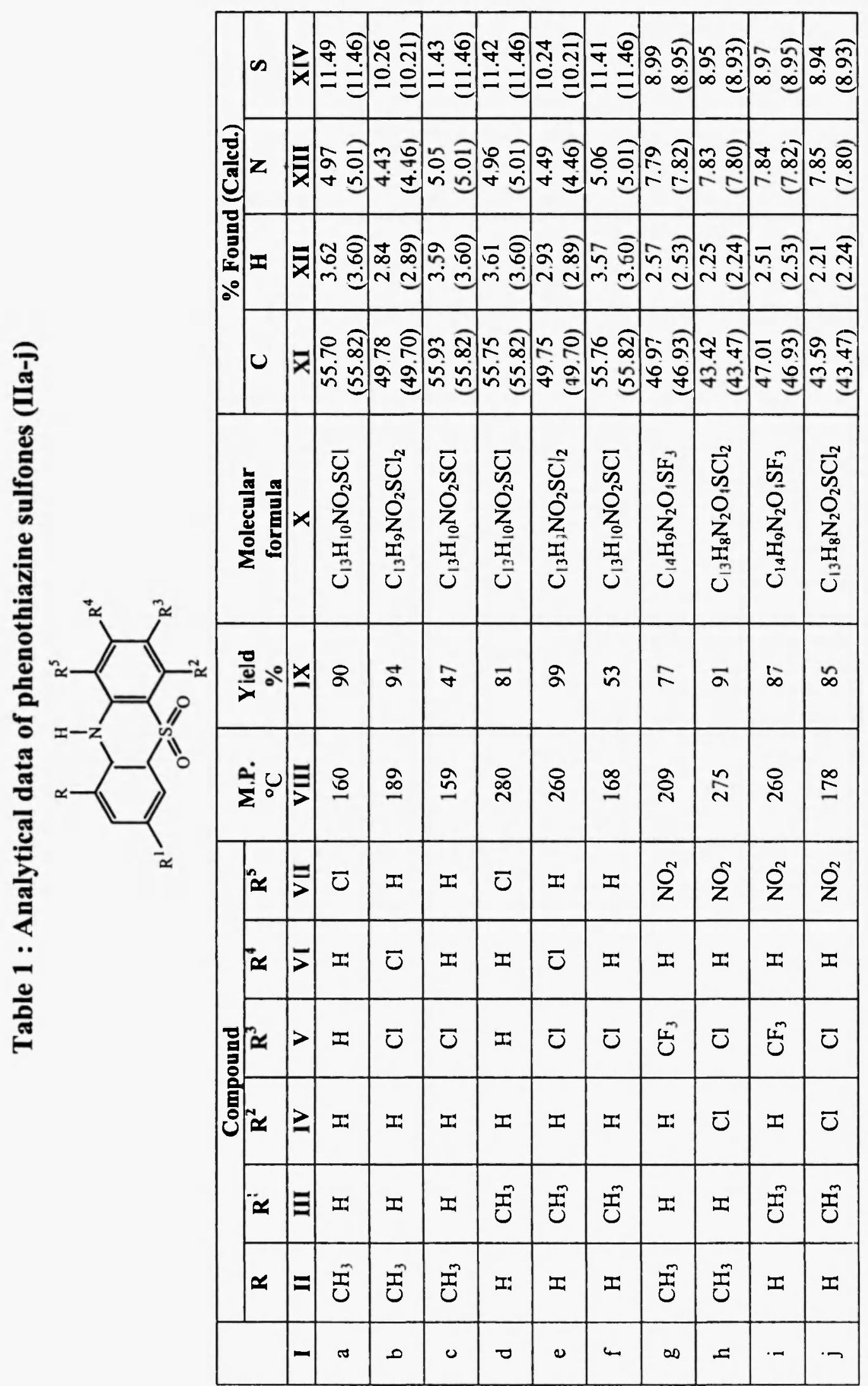




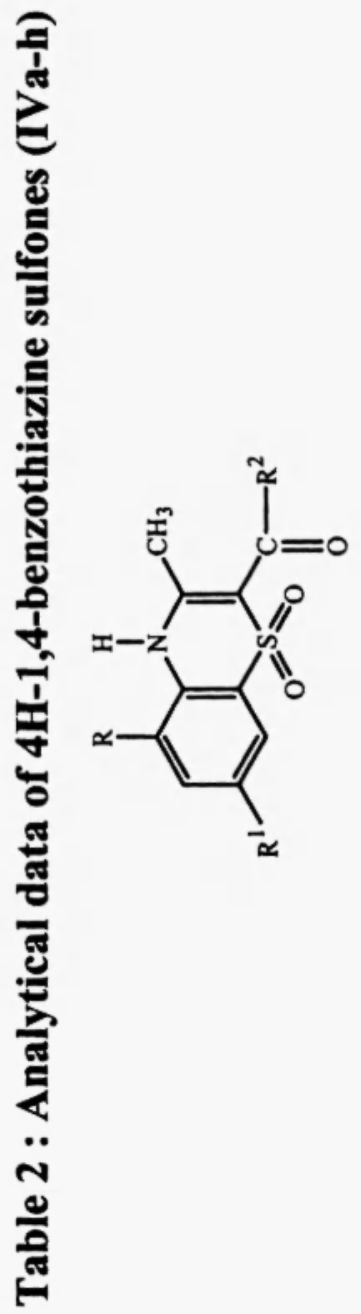

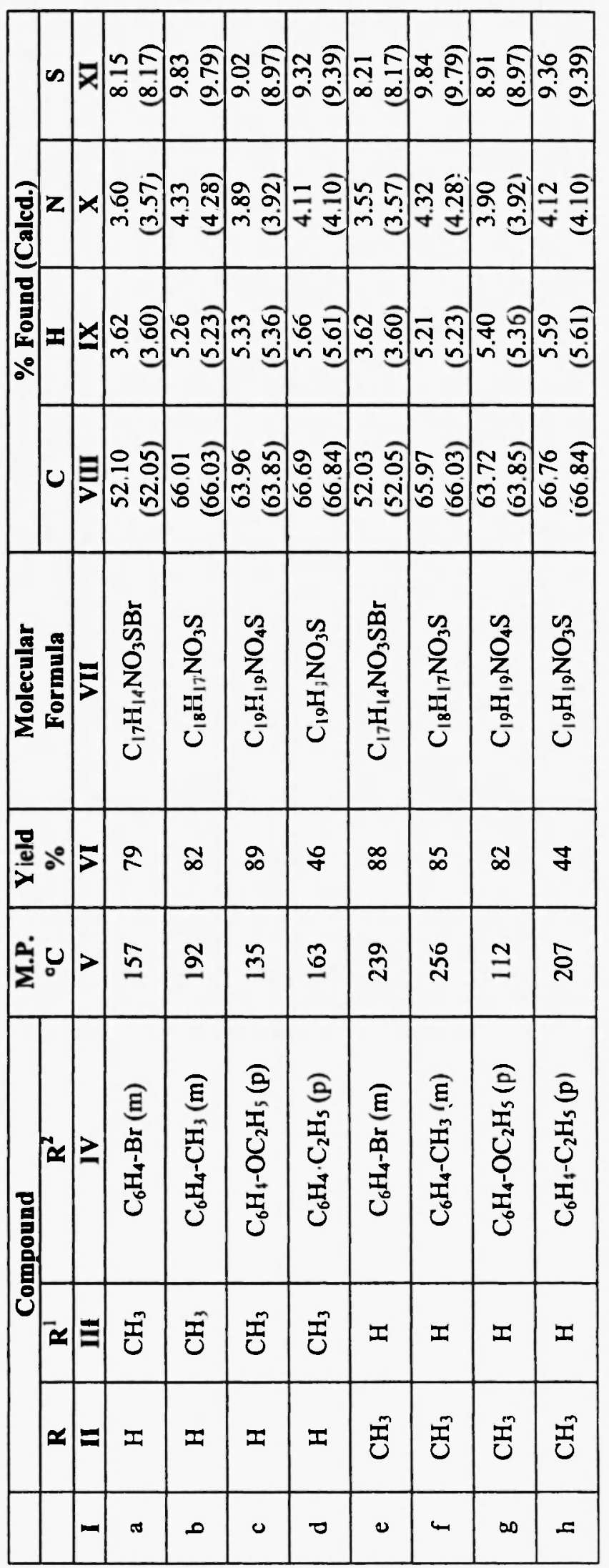


containing crushed ice. The residue separated out was collected and crystallization from ethanol afforded the desired product.

\section{Acknowledgement}

Head of the Chemistry Department, University of Rajasthan, Jaipur is thanked for providing necessary facilities. Thanks are also due to RSIC, Lucknow for providing NMR spectra.

\section{References}

1. R.R. Gupta (Ed.) "Phenothiazines and 1,4-Benzothiazines-Chemical and Biomedical Aspects", Elsevier, Amsterdam, 1988.

2. S. Shinji and A. Koshiro, Biol. Pharm. Bull., 18(4), 586-92 (1995).

3. Z. Szule, J. Mlochow and J. Palus, J. Prakt. Chem., 330(6), 1023-29 (1988).

4. T.P. Culbertson, J. Heterocycl. Chem., 28(7), 1701-03 (1991).

5. S.R. El-Ezbawy and M.A. Alshaikh, J. Chem. Technol. Biotechnol., 47(3), 209-18 (1990); Chem. Abstr., 113, 59071 (1990).

6. M. Bogusz and Moutran Wu, J. Anal. Toxicol., 15(4), 188-97 (1991); Chem. Abstr., 115, 107963 (1991).

7. A. Tureant, A.P. Cabie, A. Cailleux and P. Allain, Clin. Chem., 37(7), 1210-15 (1991); Chem. Abstr., 115, 149643 (1991).

8. J.M. Varga, G. Kalchschmid, G.F. Klein and P. Fritseh, Mol. Immunol., 28(6), 641-54 (1991); Chem. Abstr., 117, 277 (1992).

9. S. Sato and A. Koshiro, Biol. Pharm. Bull., 18(4), 586-92 (1995); Chem. Abstr., 123, 131970 (1995).

10. K.H. Mayer and A. Haberkom, German Offen. 2, 020, 298 (1971); Chem. Abstr., 76, 72533 (1972).

11. G. Filacchioni, V. Nacci and G. Stfanrich, Farmaco. Ed. Sci., 31, 478 (1996); Chem. Abstr., 85, 143048 (1976).

12. G. Fengler, D. Arlt, K. Groche, H. J. Zeiler and K. Metzger, German Offen. 3, 229, 125 (1984); Chem. Abstr., 101, 7176 (1984).

13. G. Fengler, D. Arlt and K. Groche, German Offen, 3, 329, 124 (1984); Chem. Abstr., 101, 90953 (1984).

14. R. Aki and S. Kito, Japan Kokai, 78, 32, 742 (Cl. CO3 C5/O6), 28 Mar. 1978; Chem. Abstr., 89, 207269 (1979).

15. C.R. Rasmussen, U.S. 3, 476, 749 (1969); Chem. Abstr., 72, 21727 (1970).

16. R.R. Gupta, Rakesh Kumar, G.S. Kalwania and R.K. Gautam, Ann. Soc. Sci. Bruxelles, 98(4), 195 (1984); Chem. Abstr., 106, 196375 (1987).

17. R.R. Gupta, S.K. Jain, N.K. Goswami and G.S. Kalwania, Heterocycles, 14, 831 (1980).

18. M.Y. Hamadi, R. Gupta and R.R. Gupta, Heterocycl. Commun., 4, 277 (1998).

19. M. Kumar, N. Sharma, R. Gupta and R.R. Gupta, Heterocycl. Commun., 4, 187 (1998). 